\title{
Data Analysis on the Advantages of the LEDS with Polarization-Matched Quantum
}

\author{
Ying Dai, Shanyu Quan* \\ School of Science, Shenyang University of Technology, Shenyang, China
}

\section{Email address:}

shyquan@126.com (Shanyu Quan)

\section{To cite this article:}

Ying Dai, Shanyu Quan. Data Analysis on the Advantages of the LEDS with Polarization-Matched Quantum. International Journal of Energy and Power Engineering. Vol. 4, No. 6, 2015, pp. 353-357. doi: 10.11648/j.ijepe.20150406.14

\begin{abstract}
Advantages of near-ultraviolet light-emitting diodes with polarization-matched InGaN/AlGaInN multi-quantum wells (QWs) are investigated numerically. Simulation results show that, the polarization-matched structure possesses improved efficiency droop and higher output power under high current injection due to better overlap of electron-hole wave functions and slighter Auger recombination. The optical performance of the polarization-matched structure can benefit from the enhanced capability of carrier confinement with the employment of larger bandgap electron-blocking layer and wider QWs.
\end{abstract}

Keywords: Near-Ultraviolet LEDs, Efficiency Droop, Auger Recombination

\section{具有极化匹配量子阱发光器件优势的数字研究}

\section{代颖, 权善玉 ${ }^{*}$}

理学院物理系, 沈阳工业大学, 沈阳, 中国

\section{邮箱}

shyquan@126.com（权善玉）

摘要：本文对具有极化匹配量子阱发光器件的优势进行了数字模拟。模拟结果表明在高电流下，极化匹配量子阱结构 拥有改善效率降低和提高输出功率的特点, 这主要由于该结构可以使得量子阱中的电子和空穴波函数能有效重叠, 从 而减小了俄歇复合。具有极化匹配量子阱发光器件由于采用禁带宽度大的量子阱与电子阻挡层, 从而提高了量子阱对 载流子的限域能力, 提高器件的光学性能。

关键词: 近紫外发光二极管, 效率降低, 俄歇复合

\section{1. 简介}

氮化镓基发光二极管被广泛应用在通讯产业、装饰、 全色彩显示器、液晶的背景光源以及固态照明等 $[1-6]$ 。 然而氮化镓基发光二极管自发现以来, 一直饱受 efficiency droop 的困扰 [7-11], 这阻碍氮化镓基发光 二极管在一些需要高操作电流的应用。M. H. Kim 和M. F. Schbert等学者研究指出氮化物严重的极化效应是导致 efficiency droop 的主要成因之一, 并指出采用极化匹
配 $\mathrm{A} 1 \mathrm{GaInN}$ 量子阱障可以有效降低 efficiency droop [12]。同时也研究采用反向p型氮镓铝电子阻挡层, 有效抑制了漏电流 $[13]$ 。

近紫外发光二极管由于具有高的显色指数和色稳定 性, 近年来, 被广泛应用于激发红、绿、蓝三色苂光粉以 获得白光。然而, 由于铟含量较低的氮化铟镓量子阱与氮 化镓井障的能带差较小, 载流子不易被局限在多重量子阱 的活性区内, 导致低发光功率的出现。采用禁带宽度较大 的氮化镓铝作为量子阱障可以使得量子阱比较深 [14], 可 以使更多的载流子局限在阱中, 但是氮化镓铝和氮化镓的 
晶格严重不匹配, 会存在强烈的极化电场导致能带产生倾 斜, 电子和空穴波函数重叠率降低, 使得载流子无法有效 辐射复合。采用极化匹配的 $A 1 G a I n N$ 量子阱井障可以降 低量子限域斯达克效应, 改善电子、空穴波函数的重叠。 本文主要利用APSYS 模拟软件研究使用极化匹配的 InGaN/A1GaInN 多重量子阱结构, 并与禁带宽度较大的电 子阻挡层相搭配, 以获得极佳的电子和空穴波函数重叠率, 降低俄歇非辐射复合速率, 使器件在高操作电流下, 改善 efficiency droop的现象, 从而获得更高的输出功率。

\section{2. 模拟结构及参数}

本文所使用的LED器件结构参考Huang等学者所发表 的实验结构 [15], 其发光波长为 $385 \mathrm{~nm}$, 其器件结构为原 始结构 (Origina1)。为了对比, 选择了三个器件结构进 行模拟。四个器件的量子阱均采用禁带宽度为 $3.137 \mathrm{eV}$ 的 $\mathrm{In}_{0.07} \mathrm{Ga}_{0.93} \mathrm{~N}$ 。原始器件中, 井障采用 $\mathrm{GaN}$, 选择禁带宽度 为 $3.710 \mathrm{eV}$ 的 $\mathrm{Al}_{0.16} \mathrm{Ga}_{0.84} \mathrm{~N}$ 为电子阻挡层 ( $\left.\mathrm{EBL}\right)$;

器件A: 井障采用 $\mathrm{GaN}$, 选择禁带宽度较大的 $\mathrm{Al}_{0.28} \mathrm{Ga}_{0.72} \mathrm{~N}$ 为EBL, ; 器件 $\mathrm{B}$ : 四元氮化物 $\mathrm{Al}_{0.13} \mathrm{Ga}_{0.75} \mathrm{In}_{0.12} \mathrm{~N}$ 为井障, 主要 是为了能与量子阱 $\mathrm{In}_{0.07} \mathrm{Ga}_{0.9}{ }_{3} \mathrm{~N}$ 相匹配, 而 $\mathrm{EBL}$ 与器件 $\mathrm{A}$ 相同; 器件 $\mathrm{C}$ : 电子阻挡层采用 $\mathrm{Al}_{0.28} \mathrm{Ga}_{0.72} \mathrm{~N} \quad \mathrm{EBL}$, 井障采用 $\mathrm{Al}_{0.13} \mathrm{Ga}_{0.75} \mathrm{In}_{0.12} \mathrm{~N}$, 目的是为了优化器件性能。具体结构如 表1所示。

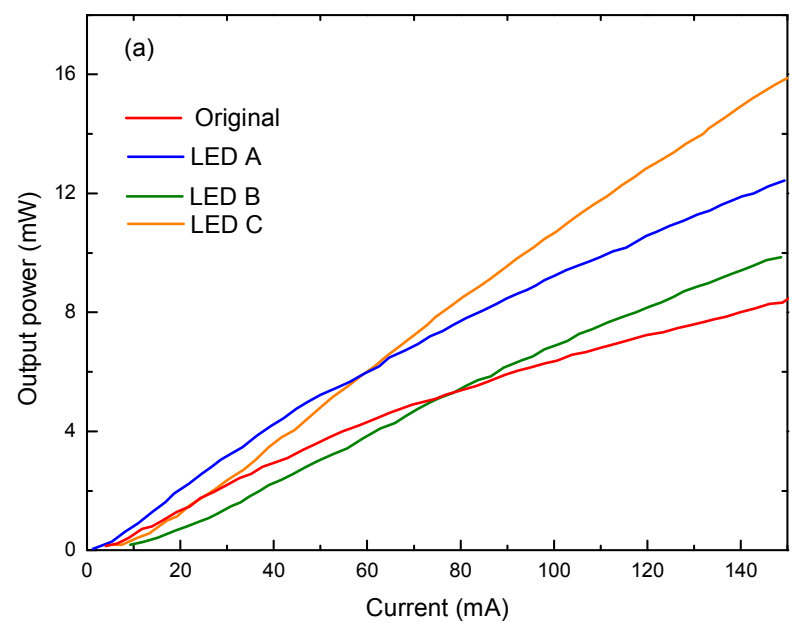

表1 器件结构。

\begin{tabular}{|c|c|c|c|c|}
\hline & Original & LED $\mathrm{A}$ & LED $\mathrm{B}$ & LED $\mathrm{C}$ \\
\hline \multirow{2}{*}{$\mathrm{p}-\mathrm{EBL}$} & $\mathrm{Al}_{0.16} \mathrm{Ga}_{0.84} \mathrm{~N}$ & $\mathrm{Al}_{0.28} \mathrm{Ga}_{0.72} \mathrm{~N}$ & $\mathrm{Al}_{0.16} \mathrm{Ga}_{0.84} \mathrm{~N}$ & $\mathrm{Al}_{0.28} \mathrm{Ga}_{0.72} \mathrm{~N}$ \\
\hline & $\mathrm{E}_{\mathrm{g}}=3.710 \mathrm{eV}$ & $\mathrm{E}_{\mathrm{g}}=3.938 \mathrm{eV}$ & $E_{\mathrm{g}}=3.710 \mathrm{eV}$ & $E_{8}=3.938 \mathrm{eV}$ \\
\hline \multirow{2}{*}{ Q B } & $\mathrm{GaN}$ & GaN & $\mathrm{Al}_{0.13} \mathrm{Ga}_{0.75} \mathrm{In}_{0.12 \mathrm{~N}} \mathrm{~N}$ & $\mathrm{Al}_{0.13} \mathrm{Ga}_{0.75} \mathrm{In}_{0.12 \mathrm{~N}} \mathrm{~N}$ \\
\hline & $E_{\mathrm{g}}=3.417 \mathrm{eV}$ & $E_{\mathrm{g}}=3.417 \mathrm{eV}$ & $\mathrm{E}_{\mathrm{g}}=3.277 \mathrm{eV}$ & $\mathrm{E}_{\mathrm{g}}=3.277 \mathrm{eV}$ \\
\hline \multirow{2}{*}{ Q W } & $\mathrm{In}_{0.07} \mathrm{Ga}_{0.93} \mathrm{~N}$ & $\mathrm{In}_{0.07} \mathrm{Ga}_{0.93} \mathrm{~N}$ & $\mathrm{In}_{0.07} \mathrm{Ga}_{0.93} \mathrm{~N}$ & $\mathrm{In}_{0.07} \mathrm{Ga}_{0.93} \mathrm{~N}$ \\
\hline & $E_{\mathrm{g}}=3.137 \mathrm{eV}$ & $E_{8}=3.137 \mathrm{eV}$ & $E_{\mathrm{g}}=3.137 \mathrm{eV}$ & $E_{8}=3.137 \mathrm{eV}$ \\
\hline
\end{tabular}

在模拟中, 背景损失和光提取率分别取为 $30 \mathrm{~cm}-1$ 和 $50 \%$, SRH寿命、辐射复合系数及俄歇复合系数分别为 $3 \mathrm{~ns}$ 、 $2 \times 10^{-11} \mathrm{~cm}^{3} \mathrm{~s}^{-1}$ 和 $4.1 \times 10^{-29} \mathrm{~cm}^{6} \mathrm{~s}^{-1}$ 。III族氮族材料系统的 禁带宽度是依据Vurgaftman 等人提出的理论模型进行的 计算 [16]。InN, GaN, 和A1N的禁带宽度取值分别为 0.641 , 3.417 和 $5.965 \mathrm{eV}[17,18]$. 能带偏差比值为 $0.7 / 0.3[19]$, 其它材料参数均依据文献 $[20]$ 。界面上因 极化而产生的极化电荷的计算依据Fiorentini 等人所提 出的方法计算而得 [21]。在理论模拟中考虑到实际器件制 备通常其极化而产生的内建电场比理论值要小, 因此模拟 中极化电荷选取了理论值的 $70 \%$ 。

\section{3. 模拟结果与分析}

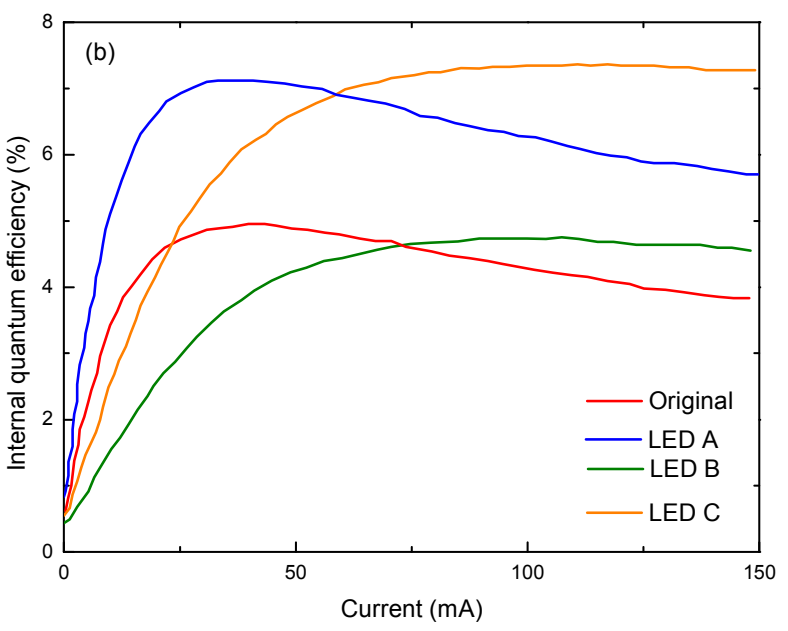

图1 四个LED器件的L-I特性图 (a) 及内部量子效率--电流关系示意图 (b)。

图1 (a) 为四个LEDs 器件的发光功率图, 在操作电流 $20 \mathrm{~mA}$ 与 $100 \mathrm{~mA}$, 对应的发光功率列于表 2 中, 模拟结果表 明, LED A 与LED C都拥有比原始结构与LED B高的发光功率, 这主要因为LED $\mathrm{A}$ 与LED C选择了具有有较大的禁带宽度的 $\mathrm{Al}_{0.28} \mathrm{Ga}_{0.72} \mathrm{~N}$ 作为 $\mathrm{EBL}$, 较高的势垒可有效降低电子溢流, 故 有较高的发光功率, 特別是在高电流100 mA下, 更明显。

表 2 在 $20 \mathrm{~mA}$ 和 $100 \mathrm{~mA}$ 下, 四个器件对应的发光功率 (单位: $\mathrm{mW}$ )。

\begin{tabular}{lllll}
\hline $\begin{array}{l}\text { Injection } \\
\text { current }\end{array}$ & Original & LED A & LED B & LED C \\
\hline $20 \mathrm{~mA}$ & 1.49 & 2.21 & 0.89 & 1.45 \\
$100 \mathrm{~mA}$ & 6.83 & 9.98 & 7.58 & 11.92 \\
\hline
\end{tabular}

图1 (b) 为四个器件的内部量子效率与电流的关系示 意图, 从图中可发现, 在低电流下, LED A有较高的内部 量子效率, 在高电流下, LED B 与LED C 可有效地抑制 efficiency droop现象, 这归功于采用极化匹配的多量子 井MQW 设计。尤其是LED C 的结构设计相较于其他三者, 确实可得到较佳的发光功率与內部量子效率。

图2为 $100 \mathrm{~mA}$ 下, LED A和LED C 器件的活性区附近的能 带图, 这两种器件结构差别在井障的不同, LED A 的井障 为禁带宽度为 $3.417 \mathrm{eV}$ 的 GaN, 而LED C 器件是采用禁带宽 度为 $3.277 \mathrm{eV}$ 的四元系 $\mathrm{Al}_{0.13} \mathrm{Ga}_{0.75} \mathrm{In}_{0.12} \mathrm{~N}$ 作为量子阱的井障。 由能带图可清楚看到, 禁带宽度较小的 $\mathrm{Al}_{0.13} \mathrm{Ga}_{0.75} \mathrm{In}_{0.12} \mathrm{~N}$ 井 障会形成较浅的量子阱, $\mathrm{In}_{0.07} \mathrm{Ga}_{0.93} \mathrm{~N} / \mathrm{GaN}$ 与 
$\mathrm{In}_{0.07} \mathrm{Ga}_{0.93} \mathrm{~N} / \mathrm{Al}_{0.13} \mathrm{Ga}_{0.75} \mathrm{In}_{0.12} \mathrm{~N}$ 的导带的band off set ( $\Delta \mathrm{Ec}$ ) 分別为 $196 \mathrm{meV}$ 与 $98 \mathrm{meV}$, 说明很小的热能就会使电子逃 离量子阱, 量子阱中载流子减少, 不利于器件的发光效率。 值得注意的是, 将电子局限在活性区的能力不仅仅由量子 阱的深浅决定, EBL 与最后井障的势能差和介面极化效应 都会影响EBL 阻碍电子流出活性区的能力。
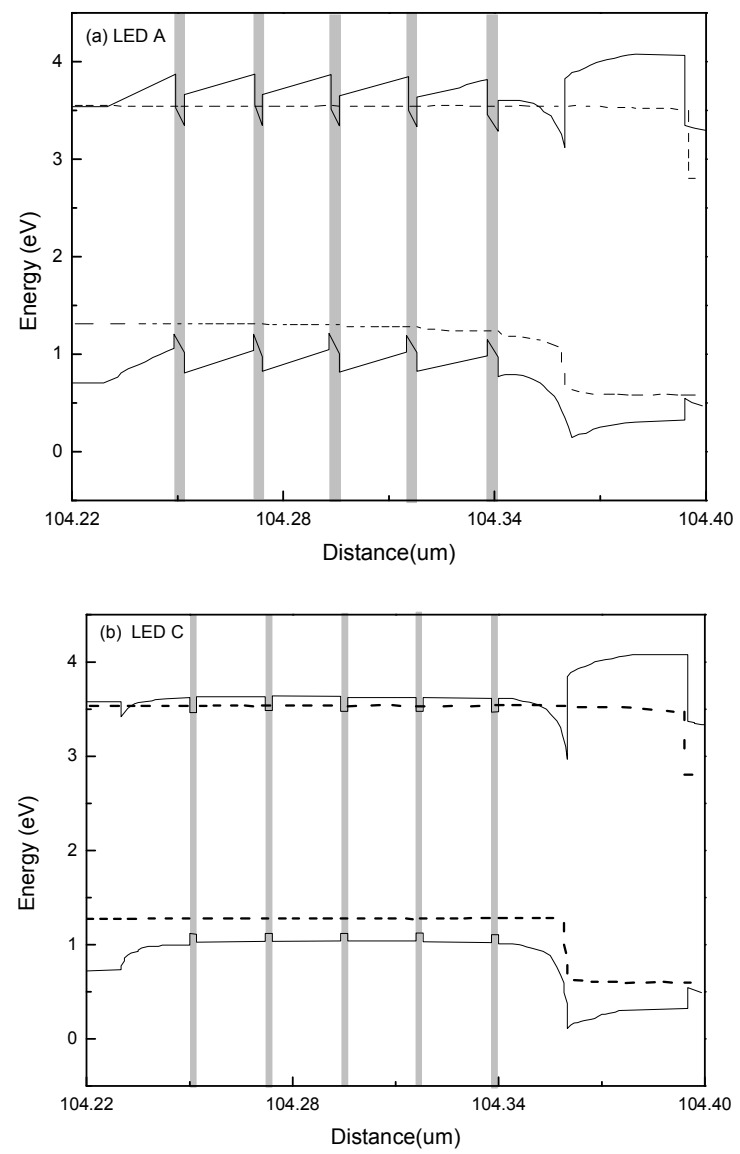

图2 100mA下, LEDS A和 LED C 器件的活性区附近的能带图。

图3给出四个器件的最后一个量子阱与EBL之间的 势能差示意图, 从图中可见, 四个器件中, 器件LED C 具 有较高的有效势垒。原始结构具有较低的势垒。同是拥 有 $\mathrm{Al}_{0.16} \mathrm{Ga}_{0.84} \mathrm{~N}$ EBL 的原始结构和 LED $\mathrm{B}$, 活性区为 $\mathrm{In}_{0.07} \mathrm{Ga}_{0 .{ }_{9}} \mathrm{~N} / \mathrm{Al}_{0.13} \mathrm{Ga}_{0.75} \mathrm{In}_{0.12} \mathrm{~N}$ 的LED B的EBL 的有效势垒 比 $\mathrm{In}_{0.07} \mathrm{Ga}_{0.93} \mathrm{~N} / \mathrm{GaN}$ 活性区设计的原始结构大, 同理, 比较 LED A 和 LED C 也有相同现象, 活性区为 $\mathrm{In}_{0.07} \mathrm{Ga}_{0.93} \mathrm{~N} / \mathrm{Al}_{0.13} \mathrm{Ga}_{0.75} \mathrm{In}_{0.12} \mathrm{~N}$ 的LED $\mathrm{C}$ 的EBL 有较高的 有效势垒。有效势垒所起的作用可从电子流密度分布情 况的图4体现出来。

从图4中可以看到, 器件LED B要比原始结构有更优秀 局限电子的能力, 显然是由于器件LED B的有效势垒比原 始结构的高的缘故。同理, LED C 相较于LED A 而言, LED $\mathrm{C}$ 的电子局限能力较好。且因极化匹配的 $\mathrm{In}_{0.07} \mathrm{Ga}_{0.93} \mathrm{~N} / \mathrm{Al}_{0.13} \mathrm{Ga}_{0.75} \mathrm{In}_{0.12} \mathrm{~N}$ 设计的LED $\mathrm{B}$ 和LED $\mathrm{C}$ 有较平 坦地的活性区能带, 故量子井中电子与空穴的波函数重叠 率极佳, 极佳的载流子波函数重叠率能有效地贡献到辐射 复合速率，有助于提升发光功率。
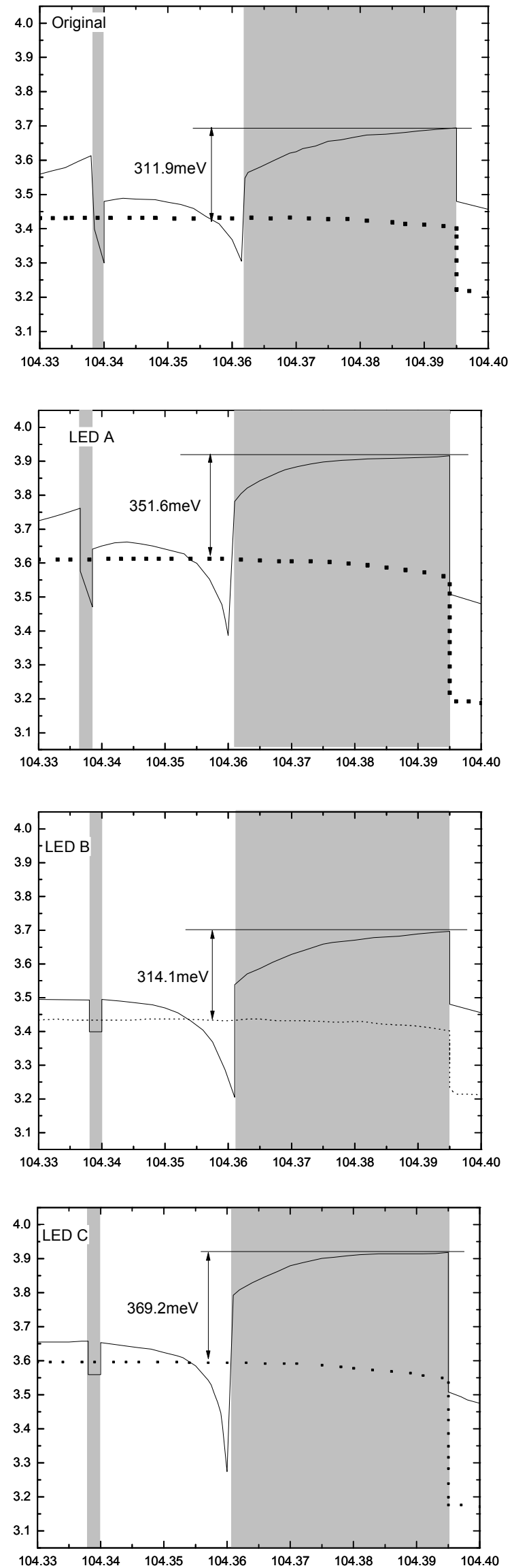

图3 四个器件最后一个量子阱与EBL的势能差。 


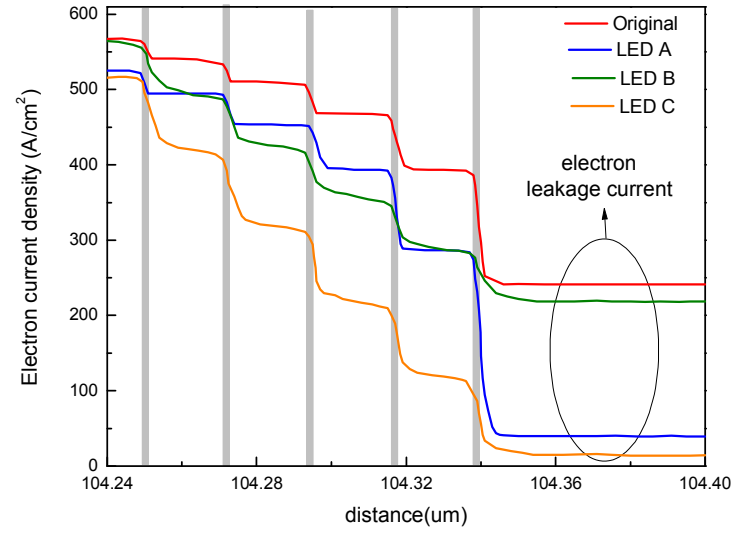

图4 四个LED器件的电子流密度分布图。

\section{4. 结论}

在紫外发光二极管中采用极化匹配的量子阱与禁带 宽度较大的EBL相搭配, 可以大大提高每个量子阱中电子 与空穴波函数的重叠率, 获得均匀的载流子分布, 提高量 子阱局限电子的能力, 有些优势尤其体现在高电流下。此 外, 极化匹配量子阱结构还可以通过增大量子阱的厚度来 消除QCSE, 减小俄歇复合。采用极化匹配量子阱结构, 尽 管量子阱较浅, 但相比原始结构仍有许多优点, 如: 降低 电子溢流, 较佳的电子与空穴波函数重叠率, 均匀的载流 子分布, 和较低的俄歇复合速率, 所以, 在发光特性上, LED B 和LED C 效率降低现象不明显, 在高电流下, 可提 升发光效率, 特别是搭配禁带宽度较大的 $\mathrm{A}_{0.28} \mathrm{Ga}_{0.72} \mathrm{~N}$ EBL 的LED C器件。此外, LED B 和LED C 的结构设计可消除 量子井中斯达克量子限域效应 (QCSE)，所以可以通过增 加量子阱的厚度, 进而提高量子阱对载流子的局限能力, 更进一步优化发光性能。

\section{参考文献}

[1] M. Koike, N. Shibata, H. Kato, and Y. Takahashi, Development of high efficiency GaN-based multiquantum-well light-emitting diodes and their applications (J) IEEE J. Sel. Topics Quantum Electron., 2002, vol. 8, pp. 271-277.

[2] C. H. Chen, S. J. Chang, Y. K. Su, J. K. Sheu, J. F. Chen, C. H. Kuo, and Y. C. Lin, Nitride-based cascade near white light-emitting diodes (J) IEEE Photonics Technol. Lett., 2002, vol. 14, pp. 908-910.

[3] A. B. Sebitosi and P. Pillay, New Technologies for Rural Lighting in Developing Countries: White LEDs (J) IEEE Trans. Energy Convers., 2007, vol. 22, pp. $674-$ 679.

[4] M. H. Crawford, LEDs for solid-state lighting: performance challenges and recent advances (J) IEEE J. Sel. Top. Quantum Electron., 2009, vol. 15, pp. $1028-1040$.
[5] E. F. Schubert and J. K. Kim, Solid-state light sources getting smart (J) Science, 2005, vol. 308, pp. $1274-1278$

[6] M. R. Krame, 0. B. Shchekin, R. Mueller-Mach, G. 0. Mueller, L. Zhou, G. Harbers, and M. G. Craford, Status and Future of High-Power Light-Emitting Diodes for Solid-State Lighting (J) J. Disp. Technol., 2007, vol. 3 , pp. $160-175$.

[7] J. -R. Chen, Y. -C. Wu, S. -C. Ling, T. - S. Ko, T. -C. Lu, H. - C. Kuo, Y. - K. Kuo, and S. - C. Wang, Auger recombination in InGaN measured by photoluminescence (J) Appl. Phys. B-Lasers Opt., 2010, vol. 98, pp. 779 -789 .

[8] H. Zhao, G. Liu, R. A. Arif, and N. Tansu, Current injection efficiency induced efficiency-droop in InGaN quantum well light-emitting diodes (J) Solid-State Electron., 2010, vol. 54, pp. 1119-1124.

[9] J. -I. Shim, D. -P. Han, H. Kim, D. -S. Shin, G. -B. Lin, D. S. Meyaard, Q. Shan, J. Cho, E. F. Schubert, H. Shim, and C. Sone, Efficiency droop in AlGaInP and GaInN light-emitting diodes (J) Appl. Phys. Lett., 2012, vol. 100, pp. 111106-1-111106-4,

[10] K. S. Kim, J. H. Kim, Y. M. Park, S. J. Jung, Y. J. Park, and S. N. Cho, Investigation of dominant effect on efficiency droop in In GaN light emitting device (J) App1. Phys. Lett., 2010, vol. 97, pp. 031113-1031113-3

[11] D. S. Meyaard, Q. Shan, J. Cho, E. F. Schubert, S. -H. Han, M. -H. Kim, C. Sone, S. J. Oh, and J. K. Kim, Temperature dependent efficiency droop in GaInN light-emitting diodes with different current densities (J) Appl. Phys. Lett., 2012, vol. 100, pp. 081106-1 - 081106-3.

[12] D. S. Meyaard, G. -B. Lin, M. Ma, J. Cho, E. F. Schubert, S. -H. Han, M. -H. Kim, H. Shim, and Y. S. Kim, Appl. Phys. Lett. 103, 201112 (2013).

[13] M. -H. Kim, M. F. Schubert, Q. Dai, J. K. Kim, E. F. Schubert, J. Piprek, and Y. Park, Origin of efficiency droop in GaN-based light-emitting diodes (J) 2007, Appl. Phys. Lett. 91, 183507-183510.

[14] M. F. Schubert, J. Xu, J. K. Kim, E. F. Schubert, M. H. Kim, S. Yoon, S. M. Lee, C. Sone, T. Sakong, and Y. Park, Polarization-matched GaInN/AlGaInN multi-quantum-well light-emitting diodes with reduced efficiency droop (J) App1. Phys. Lett. , 2008, Vol 93, 041102-041106.

[15] G. -S. Huang, H. -C. Kuo, M. -H. Lo, T. -C. Lu, J. -Y. Tsai, and S. - C. Wang, Improvement of efficiency and ESD characteristics of ultraviolet light-emitting diodes by inserting AlGaN and SiN buffer layers (J) J. Cryst. Growth , 2007, Vol 305, 55-58. 
[16] I. Vurgaftman, J. R. Meyer, and L. R. Ram-Mohan, Band parameters for III-V compound semiconductors and their alloys (J) J. Appl. Phys. , 2001, Vol 89, 5815-5819.

[17] J. Wu, W. Walukiewicz, W. Shan, K. M. Yu, J. W. Ager, S. X. Li, E. E. Haller, H. Lu, and W. J. Schaff, Temperature dependence of the fundamental band gap of InN (J) J. Appl. Phys., 2004, Vol 94, 4457-4461.

[18] N. Nepal, J. Li, M. L. Nakarmi, J. Y. Lin, and H. X. Jiang, Temperature and compositional dependence of the energy band gap of AlGaN alloys (J) Appl. Phys. Lett. , 2005, Vol 87, 242104-242108.
[19] J. Piprek and S. Nakamura, Physics of high-power InGaN/GaN lasers (J) IEE Proc. -Optoelectron. 2002, Vol 149, 145-149

[20] J. Piprek, Nitride Semicon New Technologies for Rural Lighting in Developing Countries: White LEDs Devices - Principles and Simulation (Wiley-VCH Verlag, Weinheim, 2007), p. 24-28.

[21] V. Fiorentini, F. Bernardini, and 0. Ambacher, Evidence for nonlinear macroscopic polarization in III-V nitride alloy heterostructure (J) Appl. Phys. Lett., 2002, vol. 80, pp. 1204-1206. 